\title{
New Lower Bounds on the Capacity of Optical Fiber Channels via Optimized Shaping and Detection
}

\author{
Marco Secondini Senior Member, IEEE, Stella Civelli Member, IEEE, Enrico Forestieri Senior Member, IEEE, \\ Lareb Zar Khan
}

\begin{abstract}
Constellation shaping is a practical and effective technique to improve the performance and the rate adaptivity of optical communication systems. In principle, it could also be used to mitigate the impact of nonlinear effects, possibly increasing the information rate beyond the current limit dictated by fiber nonlinearity. However, this appealing idea is frustrated by the difficulty of designing an effective shaping strategy that takes into account the nonlinearity and long memory of the fiber channel, as well as the possible interplay with other nonlinearity mitigation strategies. As a result, only little progress has been made so far, while the optimal shaping distribution and the ultimate channel capacity remain unknown. In this work, we describe a novel technique to optimize the shaping distribution in a very general setting and high-dimensional space. For a simplified block-memoryless nonlinear optical channel, the capacity lower bound obtained by the proposed technique can be expressed analytically, establishing the conditions for an unbounded growth of capacity with power. In a more realistic scenario, the technique can be implemented by a rejection sampling algorithm driven by a suitable cost function, and the corresponding achievable information rate estimated numerically. The combination of the proposed technique with an improved (non-Gaussian) decoding metric yields a new capacity lower bound for the dual-polarization WDM channel.
\end{abstract}

Index Terms-Optical fiber communication, channel capacity, achievable information rate, nonlinearity mitigation, constellation shaping.

\section{INTRODUCTION}

Modeling and mitigation of fiber nonlinearity have been an important aspect of optical fiber communication since the very beginning [2]. In the last decade, the revolution of coherent detection aided by digital signal processing (DSP) has further stimulated the research in this field, bringing two new elements to the picture: an almost unlimited capability to modulate, demodulate, and process optical signals by DSP; and the apparent impossibility to push the spectral efficiency of optical systems beyond an alleged limit set by fiber nonlinearity $[3]-[5]$. Nonetheless, many problems related to fiber nonlinearity - optimal modulation, optimal detection, and channel capacity to name a few-are still open and actively investigated.

M. Secondini, S. Civelli, E. Forestieri, and L. Zar Khan are with the Institute of Communication, Information and Perception Technologies, Scuola Superiore Sant'Anna, Pisa, Italy, and with the National Laboratory of Photonic Networks, CNIT, Pisa, Italy (email: marco.secondini@sssup.it; stella.civelli@sssup.it; forestieri@sssup.it; larebzar.khan@santannapisa.it). This paper was presented in part at the European Conference on Optical Communication, Bordeaux, France, September 2021 [1]
The problem of mitigating nonlinear effects and improving system performance has been addressed from many different perspectives. One such perspective is that of considering the optical fiber channel as a fixed element of the system, whose performance can be improved only by deploying proper DSP techniques at the transmitter or receiver. In this context, a fundamental problem is the study of the capacity of the optical fiber channel and the implementation of systems that operate at rates close to it. This problem has been widely addressed in the literature, considering different bounding techniques, fiber links, and configurations [3], [5]-[28]. For a more detailed analysis of the existing literature, we refer to [5], [29]. In this work, we focus on single-mode fibers and wavelengthdivision multiplexing (WDM) systems, studying the capacity from a single-user perspective [21]. On the one hand, the tightest available capacity lower bounds for this channel can be found by the methodologies proposed in [25], [27], which yield an achievable information rate (AIR) that reaches a peak at some optimum power and then decays. On the other hand, the only available upper bound equals the capacity of the additive white Gaussian noise (AWGN) channel and hence increases indefinitely with power [20]. The combination of these bounds does not rule out neither the existence of a finite capacity limit nor the possibility of an unbounded growth with power.

The capacity problem can be formulated in a way that entails two fundamental subproblems: the optimization of the input distribution, which is related to the implementation of an optimal coded modulation scheme; and the optimization of the decoding metric, which is related to the implementation of an optimal receiver. The maximization of the AIR obtained for a given input distribution and decoding metric yields the channel capacity. For the AWGN channel, the solution is well known. The optimal decoding metric, matched to the channel conditional distribution, factorizes into the product of marginal Gaussian distributions; analogously, the optimal input distribution factorizes into the product of identical marginal distributions-Gaussian in the general case [30], Maxwell-Boltzmann (MB) if the symbols are constrained on a given discrete alphabet [31]; the resulting AIR equals the channel capacity $C=\log _{2}(1+\mathrm{SNR}$ ) (for an unconstrained input alphabet), where SNR is the signal to noise ratio.

The picture is quite different for the nonlinear fiber channel. In this case, the conditional distribution is unknown, so that the receiver is optimized for an auxiliary channel-an 
approximated version of the true channel, for which the optimal decoding metric is available. This approach is known as mismatched decoding. Often, for simplicity and in the absence of a suitable alternative, an AWGN auxiliary channel is considered, so that the decoding metric is still taken as the product of marginal Gaussian distributions. The search for more accurate and mathematically tractable mismatched channel models is the subject of current research [32]. For instance, several models show that interchannel nonlinear interference (NLI) includes relevant phase and polarization noise (PPN) components that evolve slowly in time [33]-[35]. Such components depend also on frequency and can be alternatively represented as time-varying linear intersymbol interference [36]. Their mitigation is possible [35], [37] and yields an increase of the AIR, which is more effective if combined with subcarrier multiplexing [25], [38] and an optimized persubcarrier power allocation [26], [27]. Moreover, even the additive component of NLI has some correlation in time, which might be exploited for its mitigation [26], [27].

Constellation shaping improves the efficiency of a digital modulation scheme by modifying the position of the symbols in the constellation diagram (geometric shaping) or the frequency with which they are used (probabilistic shaping), trying to match the optimal input distribution for the given channel. A practical coded modulation scheme that has attracted much interest in recent years is probabilistic amplitude shaping (PAS), thanks to its nearly optimal performance, simple implementation, and fine rate granularity [39], [40]. PAS uses a distribution matcher, followed by a systematic forward error correction (FEC) encoder, to induce the desired distribution over a quadrature amplitude modulation (QAM) constellation. The optimal condition of i.i.d. MB symbols is approached as the block length of the distribution matcher goes to infinity [39], [41].

Constellation shaping can be used also to mitigate nonlinear effects. In this case, often referred to as nonlinear constellation shaping, the location or probability of the constellation symbols are optimized to minimize the amount or impact of the generated NLI. The main problem here is that the optimal input distribution is unknown, so that the distribution matcher approach of PAS cannot be directly implemented. There are many evidences suggesting that optimizing the marginal distribution of i.i.d. 2D symbols yields negligible gains in this case [42]. In fact, to unlock the full potentiality of nonlinear constellation shaping, the optimization should be performed in a higher dimensional space. So far, the approaches have been limited to the optimization of low-rate constellations in a low-dimensional space (e.g., geometric shaping in 4D and 8D [43], [44]), or to a highly constrained optimization of PAS in a higher-dimensional space (e.g., optimizing the block length of the distribution matcher while keeping an MB target distribution) [45]-[48]. The advantages obtained in this way are moderate, and might become negligible in the presence of carrier recovery algorithms [49].

The current research challenge is the full optimization of the constellation in a high-dimensional space, possibly in combination with improved decoding strategies. While this is an extremely complex and still unsolved problem, in this work we propose a capacity lower-bounding technique based on rejection sampling to estimate the gain achievable by such an optimization. The idea, named sequence selection, was briefly introduced in [50]. In this work, we better formalize the technique, we describe some practical procedures for its implementation, and we use it to derive some new analytical and numerical results on the optical fiber capacity.

The paper is organized as follows. Section II introduces the optical fiber channel and the capacity problem. Section III describes the proposed sequence selection technique and the related capacity lower bound. Section IV derives an analytical capacity lower bound for a simplified blockmemoryless optical channel, establishing the conditions for an unbounded growth of capacity with power. Section V presents some numerical results and the new capacity lower bound for the WDM channel. The conclusions are finally drawn in Section VI.

Notation: a random variable is denoted by an uppercase letter, e.g., $X$, its expectation by $E\{X\}$, and its realization by the corresponding lowercase letter $x$. This rule, however, is broken when dealing with some deterministic quantities, e.g., the bandwidth $W$, the power $P$, or the length $N$. The probability density function (or distribution, in short) of the random variable $X$ is simply denoted by $p(x)$, with the argument implicitly defining the specific distribution, so that $p(x)$ and $p(y)$ denote the different distributions of the variables $X$ and $Y$. A subscript is sometimes used to distinguish some specific distributions, e.g., the unbiased distribution $p_{u}(x)$. A discrete-time stochastic process is denoted by a boldface uppercase letter, e.g., $\boldsymbol{X}=\left(X_{1}, X_{2}, \ldots\right)$, and the notation $\boldsymbol{X}_{k}^{n}=\left(X_{k}, X_{k+1}, \ldots, X_{n}\right)$ and $\boldsymbol{X}^{n}=\left(X_{1}, X_{2}, \ldots, X_{n}\right)$ is used to denote finite portions of the process, i.e., finite-length random sequences. The corresponding realizations are denoted by boldface lowercase letters. The energy of the sequence $\boldsymbol{x}^{n}$ is $\left\|\boldsymbol{x}^{n}\right\|^{2}=\sum_{i=1}^{n}\left|x_{i}\right|^{2}$. Integrals, if not otherwise specified by explicit limits, extend to the whole space in which the integration variable is defined.

\section{SySteM DESCRIPTION AND PROBLEM FORMULATION}

The goal of this work is to study the capacity of the optical fiber channel, from the perspective of a single WDM user in a WDM configuration. Here the optical fiber channel is defined as a waveform channel, in which the propagation of the optical signal is governed by the Manakov equation [51]. The Manakov equation accounts for attenuation, dispersion, and Kerr nonlinearity, with the addition of a noise term and a periodic gain/loss function that accounts for the presence of optical amplifiers [25], [29].

The available optical bandwidth is divided into several independent slots of size $W$, each allotted to a different WDM user. We assume fair and independent WDM users, meaning that the multiplexed signals are independently modulated by each user, have the same input power and statistical properties, and are independently detected (behavioral model $c$ in [21]). We assume that the input and output signals of each user are strictly band-limited, with passband bandwidth $W$. In this case, with no loss of generality, it is possible to give an 


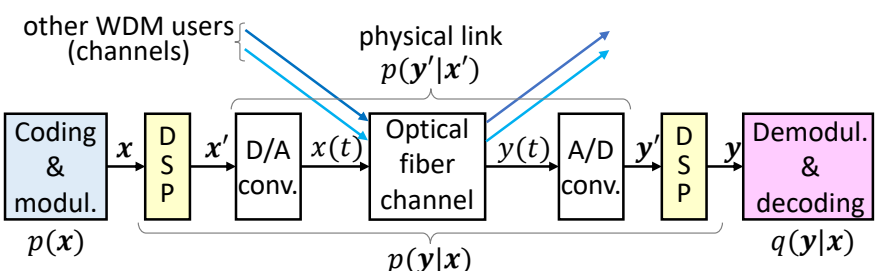

Fig. 1. Description of the system considered for AIR computation and maximization.

equivalent discrete-time formulation of the problem, where the input and output signals are represented by their samples taken at Nyquist rate $W$. The sampling theorem [30], [52] ensures that the input and output samples are a sufficient statistic to represent the input and output waveforms, respectively, so that any possible coding and decoding strategy for the waveform channel can be implemented by working on the input and output samples.

From a single-user perspective, the system is described as in Fig. 1. At the transmitter, a random message (a sequence of independent uniformly distributed bits) is encoded on a sequence of symbols $\boldsymbol{x}=\left(x_{1}, x_{2}, \ldots\right)$ by a proper combination of coding and modulation. The input sequence $\boldsymbol{x}$ is processed by a digital signal processing (DSP) block to obtain the sequence $\boldsymbol{x}^{\prime}=\left(x_{1}^{\prime}, x_{2}^{\prime}, \ldots\right)$ and converted to the input waveform $x(t)$ by an ideal digital-to-analog (D/A) converter with sampling rate and bandwidth $W$. After propagation of $x(t)$ through the optical fiber channel, the output waveform $y(t)$ is converted into the sequence $\boldsymbol{y}^{\prime}$ by an ideal analog-todigital (A/D) converter with bandwidth and sampling rate $W$ and digitally processed to obtain $\boldsymbol{y}$. The transmitted message is finally recovered from $\boldsymbol{y}$ by demodulation and decoding.

For every $N=1,2, \ldots$, the combination of coding and modulation is characterized by the distribution $p\left(\boldsymbol{x}^{N}\right)$ of the emitted sequence; the discrete-time physical link-obtained by the combination of D/A converter, optical fiber link, and A/D converter-is characterized by the conditional distribution $p\left(\boldsymbol{y}^{\prime N} \mid \boldsymbol{x}^{\prime N}\right)$; and the combination of demodulation and decoding is characterized by the decoding metric $q\left(\boldsymbol{y}^{N} \mid \boldsymbol{x}^{N}\right)$. Usually, DSP is employed at the transmitter and/or receiver to compensate for some channel impairments (e.g., fiber dispersion) and to perform some important tasks such as clock and carrier recovery. The combination of DSP and discretetime physical link gives the overall discrete-time channel $p\left(\boldsymbol{y}^{N} \mid \boldsymbol{x}^{N}\right)$.

The system performance is measured by the achievable information rate (AIR) with mismatched decoding (also known as auxiliary-channel lower bound) [53]

$$
I_{q}(\boldsymbol{X} ; \boldsymbol{Y})=\lim _{N \rightarrow \infty} \frac{1}{N} E\left\{\log _{2} \frac{q\left(\boldsymbol{Y}^{N} \mid \boldsymbol{X}^{N}\right)}{q\left(\boldsymbol{Y}^{N}\right)}\right\}
$$

where

$$
q\left(\boldsymbol{y}^{N}\right)=\int q\left(\boldsymbol{y}^{N} \mid \boldsymbol{x}^{N}\right) p\left(\boldsymbol{x}^{N}\right) d \boldsymbol{x}^{N}
$$

is the mismatched output distribution, obtained by connecting the input source to an auxiliary channel with conditional distribution $q\left(\boldsymbol{y}^{N} \mid \boldsymbol{x}^{N}\right)$; the limit operation accounts for channel memory; and the expectation is taken with respect to the input distribution $p\left(\boldsymbol{x}^{N}\right)$ and true channel distribution $p\left(\boldsymbol{y}^{N} \mid \boldsymbol{x}^{N}\right)$. In practice, (1) can be estimated by

$$
I_{q}(\boldsymbol{X} ; \boldsymbol{Y}) \approx \frac{1}{N} \log _{2} \frac{q\left(\boldsymbol{y}^{N} \mid \boldsymbol{x}^{N}\right)}{q\left(\boldsymbol{y}^{N}\right)}
$$

taking $N$ sufficiently long to ensure that both the limit and expectation operations are approximated with the desired accuracy.

The AIR (1) can be converted into a spectral efficiency (SE) by dividing it by the product $W T$, where $W$ is the channel bandwidth and $T$ the symbol rate. The choice $T=1 / W$ made above means that the AIR values obtained in this work (in bits/symbol) can be directly read as SE values (in bits/s/Hz).

Single-mode fibers allow the propagation of two orthogonal polarization modes. For the sake of simplicity, this is not explicitly considered in Fig. 1 and in the notation employed in the paper, where symbols and signals are defined in $\mathbb{C}(2 \mathrm{D}$ space). In fact, when a WDM user exploits both modes-as usual in modern coherent systems-the corresponding symbols and signals should be considered as dual-polarization complex symbols and signals, defined in the 4D space $\mathbb{C}^{2}$. In this case, the AIR in (1) is expressed in bits/4D symbol. This specific scenario is considered in Sections $\mathrm{V}-\mathrm{C}$ and $\mathrm{V}-\mathrm{D}$, where the numerical results are anyway reported in bits/2D symbol and bits/s/Hz/polarization by simply dividing the AIR by two.

The capacity problem studied in this work can be seen as an optimization problem, where the AIR (1) of the system in Fig. 1 is maximized by optimizing the input distribution $p\left(\boldsymbol{x}^{N}\right)$ (blue block), the decoding metric $q\left(\boldsymbol{y}^{N} \mid \boldsymbol{x}^{N}\right)$ (pink block), and the DSP at transmitter and receiver (yellow blocks). Any specific but suboptimal choice of these blocks yields a capacity lower bound. In practice, this work and most of the current research on this topic is focused on finding better combinations of these three elements to improve on the existing lower bounds. The choice $T=1 / W$ made above and the sampling theorem ensure that the capacity obtained in this way equals the maximal SE for the waveform channel, no further improvements being possible by changing the sampling rate.

With respect to the classical formulation of channel capacity [30], [54], [55], the optimization problem formulated above contains some additional elements-namely, the decoding metric and the DSP-which might be practically useful, though formally redundant. In fact, the optimal decoding metric is known to be $q\left(\boldsymbol{y}^{N} \mid \boldsymbol{x}^{N}\right)=p\left(\boldsymbol{y}^{N} \mid \boldsymbol{x}^{N}\right)$. However, when the true distribution $p\left(\boldsymbol{y}^{N} \mid \boldsymbol{x}^{N}\right)$ is either unavailable or too complicated (as in the case of Fig. 1), the detector is designed to make maximum-a-posteriori-probability decisions based on a simpler but mismatched channel law $q\left(\boldsymbol{y}^{N} \mid \boldsymbol{x}^{N}\right) \neq p\left(\boldsymbol{y}^{N} \mid \boldsymbol{x}^{N}\right)$ [56], which is then optimized subject to some reasonable assumptions and complexity constraints. The derivation of improved decoding metrics for the system in Fig. 1] is discussed, for instance, in [24]-[27]. Some of these metrics will be used to obtain the capacity lower bounds shown in Section V-D

For what concerns the DSP, its optimization is clearly unnecessary, since any DSP can be formally included in a 
proper definition of $p\left(\boldsymbol{x}^{N}\right)$ and $q\left(\boldsymbol{y}^{N} \mid \boldsymbol{x}^{N}\right)$, whose optimization therefore includes the DSP optimization. However, a properly designed DSP can simplify the channel $p\left(\boldsymbol{y}^{N} \mid \boldsymbol{x}^{N}\right)$ (e.g., by shortening its memory or removing some undesired effects) and the search for the corresponding optimal input distribution and decoding metric. Two practical examples that are considered in this work are chromatic dispersion compensation, which completely removes the effect of chromatic dispersion in the linear regime, and single-channel digital backpropagation, which removes deterministic intrachannel NLI, leaving interchannel NLI as a dominant impairment [13].

\section{InPut Optimization via Sequence SELEction}

\section{A. General Idea}

We first illustrate the basic idea with the simple example depicted in Fig. 2. Given a discrete-time channel, we want to find the most efficient way to encode a message of $k$ information bits on a sequence of $n$ symbols, where each symbol belongs to a given $M$-ary modulation alphabet (e.g., a 16-QAM symbol in the figure). Clearly, the problem has no solution if $R \triangleq k / n>\log _{2} M$, where $R$ is the code rate, as in this case the number of possible messages $2^{k}$ exceeds the number of available sequences $M^{n}$. On the other hand, when $R=\log _{2} M$, there is only one trivial solution, which uses the whole set $\mathcal{A}$ of available sequences (the particular mapping order being irrelevant). The problem becomes more interesting when $R<\log _{2} M$. In this case, there are more sequences than messages, meaning that we can find an optimum map that uses only the "best" $2^{k}$ sequences, contained in a subset $\mathcal{B} \subset \mathcal{A}$. This selection process entails the existence of a certain cost function, which can be used to rank the sequences according to their cost and to select the "least expensive" ones, discarding the others (e.g., the red one in Fig. 2). At this point, the most efficient encoding strategy is simply obtained by mapping each possible message to a different sequence of the subset $\mathcal{B}$ (in any arbitrary order), as shown in the table in Fig. 2 In practice, by changing the cardinality of the subset $|\mathcal{B}|=2^{k}$, we can obtain a different trade-off between code rate $R$ and average cost. For instance, by selecting less sequences, we reduce the transmission rate but also the average cost (which means, for instance, a higher energy-efficiency or a better performance, depending on the considered cost function).

\section{B. Rejection Sampling Algorithm}

The simple idea illustrated above raises several important issues. The first issue is related to the procedure used to define the subset $\mathcal{B} \subseteq \mathcal{A}$ of "best" sequences. In fact, the proposed exhaustive search becomes clearly unfeasible for large $M$ and $n$, or even impossible when considering a continuous input constellation. Here we propose a different approach, introducing a mechanism to implement an optimized source, i.e., a source that emits random sequences with an optimized input distribution. The optimization is based on the same idea presented in Section III-A, but it avoids an exhaustive search and works even in the general case of a continuous sampling space with large dimensionality.

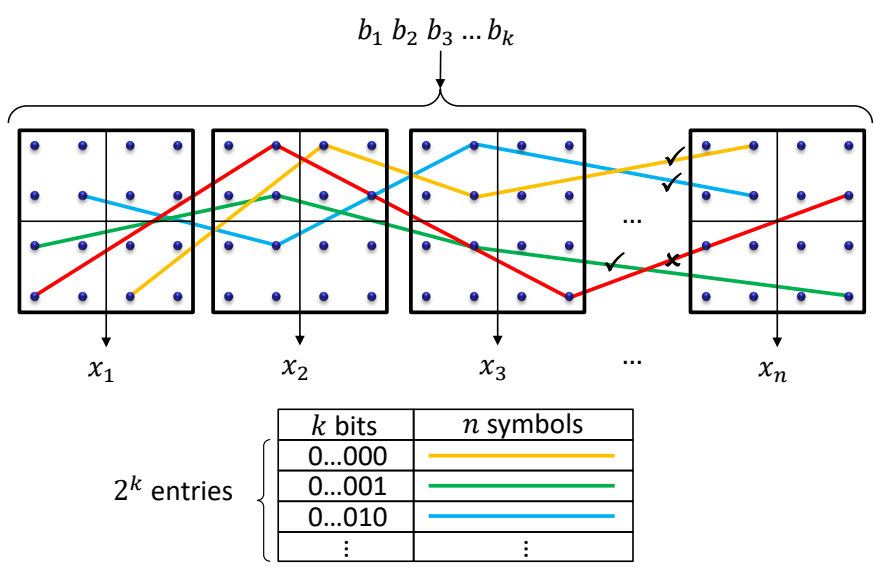

Fig. 2. The sequence selection approach: $k$ input bits are mapped to the "best" $2^{k}$ sequences of $n M$-ary symbols, with rate $R=k / n \leq \log _{2} M$.

Optimized source

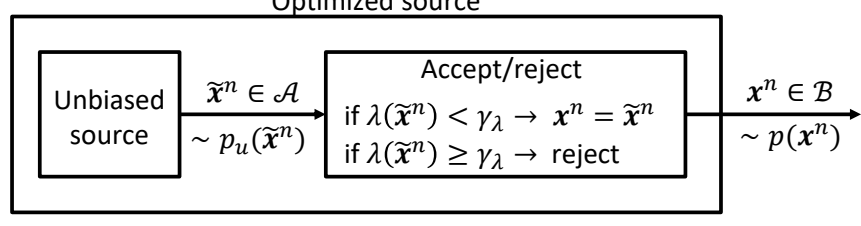

Fig. 3. The rejection sampling machine that generates sequences with the optimized distribution.

The optimized source is implemented by combining an unbiased source with an accept-reject algorithm, as shown in Fig. 3 The unbiased source draws a random sequence $\tilde{\boldsymbol{x}}^{n}$ from a certain sampling space $\mathcal{A} \subseteq \mathbb{C}^{n}$ with proposal (unbiased) distribution $p_{u}\left(\tilde{\boldsymbol{x}}^{n}\right)$. In general, both the sampling space and the proposal distribution can be arbitrarily selected, meaning that the symbols can be drawn from a discrete or continuous constellation, be i.i.d. or not, and have any distribution. In practice, it is convenient to select a sampling space that matches the hardware constraints that we have at the transmitter (e.g., the whole $\mathbb{C}^{n}$ for a theoretical analysis, the Cartesian product of $n$ QAM constellations for a practical transmitter) and a proposal distribution that is simple enough in terms of sample generation and AIR computation, but not too far from the optimal one. In this work, we will consider sequences of $n$ i.i.d. complex symbols with a circularlysymmetric Gaussian distribution.

The accept-reject algorithm is characterized by a cost (or loss) function $\lambda\left(\tilde{\boldsymbol{x}}^{n}\right)$ and a selection threshold $\gamma_{\lambda}$. The proposed sequence $\tilde{\boldsymbol{x}}^{n}$ is accepted (emitted by the optimized source by setting $\left.\boldsymbol{x}^{n}=\tilde{\boldsymbol{x}}^{n}\right)$ if $\lambda\left(\tilde{\boldsymbol{x}}^{n}\right)<\gamma_{\lambda}$, rejected otherwise. As a result, the distribution of the sequences emitted by the optimized source is

$$
p\left(\boldsymbol{x}^{n}\right)= \begin{cases}\frac{1}{\eta} p_{u}\left(\boldsymbol{x}^{n}\right), & \lambda\left(\boldsymbol{x}^{n}\right)<\gamma_{\lambda} \\ 0, & \lambda\left(\boldsymbol{x}^{n}\right) \geq \gamma_{\lambda}\end{cases}
$$

where

$$
\eta=\operatorname{Pr}\left\{\lambda\left(\tilde{\boldsymbol{X}}^{n}\right)<\gamma_{\lambda}\right\}
$$

is the acceptance probability, which can be practically estimated as $\eta \approx N_{a} / N_{p}$, where $N_{a}$ is the number of accepted 
sequences and $N_{p}$ is the number of proposed sequences. On average, each accepted sequence requires the generation of $1 / \eta$ proposed sequences and the computation of the corresponding $1 / \eta$ values of the cost function. This gives an indication of the computational cost of the proposed procedure.

In practice, the algorithm guarantees that all the emitted sequences belong to the set $\mathcal{B} \subseteq \mathcal{A}$ of "best" sequences (whose cost is lower than the prescribed threshold $\gamma_{\lambda}$ ); within such set, their distribution is just a rescaled version of the unbiased one. Reducing (increasing) $\gamma_{\lambda}$ reduces the average cost of the sequences emitted by the optimized source. At the same time, it reduces also the acceptance rate $\eta$, which in turn reduces the (differential) entropy rate of the optimized source (in the example of Fig. 2, the number of sequences $|\mathcal{B}|=2^{k}$ available to encode information decreases, reducing the rate $k / n)$ and increases the computational complexity of the generation procedure.

\section{AIR Estimation}

In principle, the AIR with mismatched decoding and the optimized source described in Section III-B can be estimated by the general expression in 11 , where the input sequence $\boldsymbol{x}^{N}$ is obtained by concatenating several independent subsequences of length $n$ emitted by the optimized source (for simplicity, we assume that $N / n$ is an integer number). The corresponding input distribution $p\left(\boldsymbol{x}^{N}\right)$ is hence the product of the distributions of the $N / n$ subsequences, given by (4), while the mismatched output distribution $q\left(\boldsymbol{y}^{N}\right)$ is given by 22. However, since 4 is a complicated distribution in an $n$-dimensional space, the computation of (2) can be very complex in this case. Thus, we resort to the lower bound

$$
\begin{aligned}
I_{\eta}(\boldsymbol{X} ; \boldsymbol{Y})=\lim _{N \rightarrow \infty} \frac{1}{N} E\left\{\log _{2} \frac{q\left(\boldsymbol{Y}^{N} \mid \boldsymbol{X}^{N}\right)}{q_{u}\left(\boldsymbol{Y}^{N}\right)}\right\} \\
-\frac{1}{n} \log _{2} \frac{1}{\eta} \leq I_{q}(\boldsymbol{X} ; \boldsymbol{Y})
\end{aligned}
$$

where the expectation is taken with respect to the actual optimized distribution; the inequality is obtained by using $p\left(\boldsymbol{x}^{N}\right) \leq p_{u}\left(\boldsymbol{x}^{N}\right) / \eta^{N / n}$, which follows from 4 ; ; and

$$
q_{u}\left(\boldsymbol{y}^{N}\right)=\int q\left(\boldsymbol{y}^{N} \mid \boldsymbol{x}^{N}\right) p_{u}\left(\boldsymbol{x}^{N}\right) d \boldsymbol{x}^{N}
$$

is obtained by connecting the unbiased source $p_{u}\left(\boldsymbol{x}^{N}\right)$ to the auxiliary channel $q\left(\boldsymbol{y}^{N} \mid \boldsymbol{x}^{N}\right)$ and is, hence, much simpler to compute than $q\left(\boldsymbol{y}^{N}\right)$. For instance, for an unbiased source of i.i.d. Gaussian samples with variance $P$ and an AWN decoding metric with variance $\sigma^{2}$, the samples at the output of the auxiliary channel are i.i.d. Gaussian with variance $P+\sigma^{2}$. In practice, the AIR (6) can be estimated by the following simple procedure:

- create a long input sequence $\boldsymbol{x}^{N}$ by concatenating $N / n$ subsequences of length $n$ generated by the rejection sampling machine in Fig. 3 ,

- compute numerically (or generate experimentally) the corresponding output sequence $\boldsymbol{y}^{N}$ obtained by the propagation of $\boldsymbol{x}^{N}$ through the system in Fig. 1.
- estimate the AIR using

$$
I_{\eta}(X ; Y) \approx \frac{1}{N} \log _{2} \frac{q\left(\boldsymbol{y}^{N} \mid \boldsymbol{x}^{N}\right)}{q_{u}\left(\boldsymbol{y}^{N}\right)}-\frac{1}{n} \log _{2} \frac{N_{p}}{N_{a}}
$$

i.e., using the same expression as in the unbiased case and subtracting the rate loss.

The AIR can then be maximized by optimizing the selection threshold $\gamma_{\lambda}$, i.e., the acceptance rate $\eta \approx N_{a} / N_{p}$. The overall procedure is very similar to the case without sequence selection, the only additional complexity being the generation of the input symbols based on the rejection sampling machine in Fig. III-B. which requires $1 / \eta$ computations of the cost function for each accepted subsequence of length $n$.

\section{Cost Function}

In practice, the definition of a suitable cost function is essential for the correct optimization of the source. A good cost function must be simple enough to be computed several times per each generated sequence and, at the same time, accurate enough to yield an effective selection. The final goal is that of maximizing the AIR (1) over the given channel, subject to some possible constraints. This means that, in general, the cost function should be specifically defined to account for the channel characteristics, the decoding metric, and the required constraints.

As a first example, we consider a simple AWGN channel with matched detection and an average-power constraint. In this case, the channel effect is independent of the particular input sequence and depends only on the noise variance. On the other hand, different sequences may have a different energy, meaning that their use may have a different cost in terms of average power. Therefore, given a generic input sequence $s^{n}$, a suitable cost function appears to be the energy per symbol of the sequence

$$
\lambda\left(s^{n}\right)=\frac{1}{n}\left\|s^{n}\right\|^{2}
$$

With reference to the example in Fig. 2, setting a particular threshold $\gamma_{\lambda}$ for the cost function in $(9)$ corresponds to select all the sequences within the sphere of radius $\sqrt{n \gamma_{\lambda}}$ in $\mathbb{C}^{n}$. Changing the threshold $\gamma_{\lambda}$ changes the number of selected sequences $2^{k}$, i.e., the rate $R=k / n$ of the shaping strategy. Among all possible strategies, the sphere shaping induced by the cost function (9) clearly minimizes the average power required to achieve the desired rate.

The situation is different for the nonlinear channel, which induces also signal-dependent distortions due to fiber nonlinearity. At the same time, the average power constraint might be no longer relevant in this case, since the AIR has typically a peaky behavior and the goal is that of maximizing the peak AIR, regardless of the average power. The impact of the channel on the AIR (6) is determined by the (mismatched) conditional entropy term $-E\left\{\log _{2} q\left(\boldsymbol{Y}^{N} \mid \boldsymbol{X}^{N}\right)\right\} / N$, which measures the average uncertainty that we have about the output sequence, given the input sequence and the mismatched 
probabilistic model $q\left(\boldsymbol{y}^{N} \mid \boldsymbol{x}^{N}\right) 1$ Therefore, a suitable cost function to minimize this term (and maximize the AIR) is

$$
\left.\lambda\left(s^{n}\right)=-\frac{1}{N} E\left\{\log _{2} q\left(\boldsymbol{Y}^{N} \mid \boldsymbol{X}^{N}\right) \mid \boldsymbol{X}_{N / 2-n / 2+1}^{N / 2+n / 2}=s^{n}\right)\right\}
$$

which measures the average effect that the particular sequence $s^{n}$ has on the mismatched conditional entropy term. Note that, in the case of an AWGN channel with matched decoding, the cost function (10) equals the entropy of the noise and is independent of the sequence $s^{n}$, meaning that all the sequences are equally good. In this case, the introduction of an average power constraint and the use of the cost function (9) is clearly more appropriate.

It is instructive to specialize the cost function above to the case of an AWGN decoding metric

$$
q\left(\boldsymbol{y}^{N} \mid \boldsymbol{x}^{N}\right)=\frac{1}{\pi \sigma^{2 N}} \exp \left(-\frac{\left\|\boldsymbol{y}^{N}-\boldsymbol{x}^{N}\right\|^{2}}{\sigma^{2}}\right)
$$

By replacing (11) in (10) and omitting some inessential constant terms (which do not alter the ranking induced by the cost function over the available sequences), the cost function can be expressed as

$$
\lambda\left(s^{n}\right)=\frac{1}{N} E\left\{\left\|\boldsymbol{Y}^{N}-\boldsymbol{X}^{N}\right\|^{2} \mid \boldsymbol{X}_{N / 2-n / 2+1}^{N / 2+n / 2}=s^{n}\right\}
$$

In practice, 12 can be estimated by replacing the expectation with an average over a finite number of realizations, and considering only a shorter portion of the sequence, i.e., only those symbols which are more affected by the input sequence $s^{n}$ - the $n$ central symbols and, possibly, a few surrounding ones, depending on the channel memory.

A final simplification can be obtained by assuming that nonlinear effects are block-memoryless, with blocklength $n$, and independent of the noise. In this case, both the noise and the symbols outside the block of length $n$ become irrelevant, as they contribute only with a constant term to the cost function (12), which can therefore be simplified as

$$
\lambda\left(\boldsymbol{s}^{n}\right)=\frac{1}{n}\left\|\hat{\boldsymbol{y}}^{n}-\boldsymbol{s}^{n}\right\|^{2}
$$

where the vector $\hat{\boldsymbol{y}}^{n}$ collects the $n$ output symbols obtained from a noiseless propagation of the input sequence $\boldsymbol{x}^{n}=\boldsymbol{s}^{n}$. The block-memoryless assumption ensures that $\hat{\boldsymbol{y}}^{n}$ depends deterministically on $s^{n}$, so that the expectation is no longer required and the cost function can be estimated from a single noiseless simulation.

\section{Analytical Results}

We apply here the proposed technique to a simple nonlinear channel that includes AWGN and block-memoryless NLI.

\footnotetext{
${ }^{1}$ Some authors use the term "cross entropy" to refer to the quantity $-E_{p}\{\log q(X)\}$, where $p(x)$ and $q(x)$ are two distributions defined over the same probability space, and $E_{p}\{\cdot\}$ denotes expectation with respect to $p$. The same term is used by other authors to refer to the relative entropy or Kullback-Leibler divergence $-E_{p}\{\log (q(X) / p(X))$ [55]. To avoid confusion and better highlight the relation with the mismatched probabilistic model, we prefer here the term "mismatched entropy".
}

Given a block of $n$ input symbols $\boldsymbol{x}^{n}$, the corresponding output block can be written as

$$
\boldsymbol{y}^{n}=\boldsymbol{x}^{n}+\boldsymbol{w}^{n}+\boldsymbol{\xi}^{n}
$$

where $\boldsymbol{w}^{n}$ is a vector of i.i.d. noise samples with circularly symmetric complex Gaussian distribution with variance $\sigma_{w}^{2}$, and $\boldsymbol{\xi}^{n}$ is a vector of NLI samples. As in a regularperturbation-based fiber channel model [16], [34], we assume that NLI samples are generated by the nonlinear interaction of the input symbols during propagation and scale cubically with them, so that their variance can be written as $\sigma_{\xi}^{2}=a P^{3}$, where $P=E\left\{\left|X_{i}\right|^{2}\right\}$ is the input power and $a$ a proportionality constant. Moreover, we assume that channel memory does not extend beyond the edges of each block, i.e., separate blocks are independent. We remark that (14), with the related assumptions, is not intended as an accurate description of a realistic fiber channel but, rather, as a simplified model that allows for an analytical study of the problem while retaining some essential features of optical fiber channels.

First, we consider the simple case in which the system is designed according to a simple Gaussian noise model, i.e., by completely neglecting the dependence of NLI on the input symbols and by assuming that the elements of $\boldsymbol{\xi}^{n}$ are i.i.d. circularly symmetric complex Gaussian variables [57]. In this case, (14) reduces to an AWGN channel with noise variance $\sigma^{2}=\sigma_{w}^{2}+\sigma_{\xi}^{2}$, for which the optimal input distribution consists in i.i.d. circularly symmetric complex Gaussian symbols, and the optimal decoding metric is given by (11). The corresponding AIR is

$$
I_{G}(X ; Y)=\log _{2}\left(1+\frac{P}{\sigma_{w}^{2}+a P^{3}}\right),
$$

which has the typical behavior shown in Fig. 4 with a red dashed line for the case $a=0.01$ and $\sigma_{w}^{2}=0.001$. With respect to the linear capacity $C=\log _{2}\left(1+P / \sigma_{w}^{2}\right)$ obtained for $a=0$, which grows unbounded with power, the Gaussian AIR (15) over the nonlinear channel reaches a peak at the optimal input power $P_{\text {opt }}=\sqrt[3]{\sigma_{w}^{2} / 2 a}$, after which it decreases again and vanishes. Though this behavior appears often in the literature with reference to the optical fiber channel or its approximated models, it has been already pointed out that there is no proof that a similar behavior applies to the actual channel capacity. Indeed, a different behavior has been demonstrated for different approximated channel models, whereas the problem remains open when considering a realistic channel [5], [29].

Then, we consider a different approach, in which we account for the dependence of NLI on the input symbols in (14) to better shape the input distribution. In particular, we optimize the distribution of the input symbols by applying the sequence selection procedure described in Section III to an unbiased source of i.i.d. Gaussian symbols with power $P$. The length of the input sequences is taken equal to the block length $n$ over which the channel memory extends. On the other hand, we do not optimize the decoding metric, keeping the simple AWGN metric in (11). For this metric, given the blockmemoryless property of the channel, a suitable cost function 


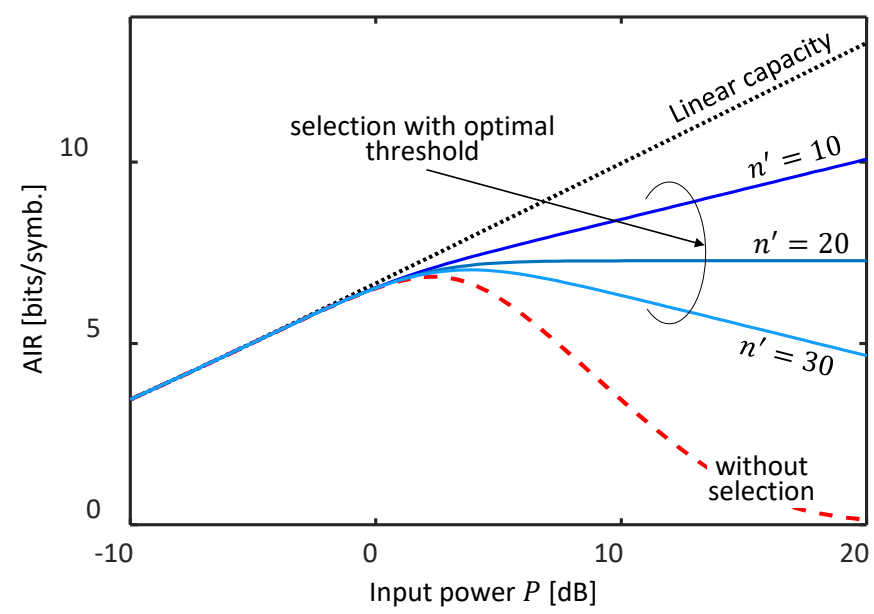

Fig. 4. AIR over the nonlinear block-memoryless channel with Gaussian decoding metric, with and without sequence selection.

is the energy per symbol of the NLI [13, which in this case can be expressed as

$$
\lambda\left(\boldsymbol{x}^{n}\right)=\frac{1}{n}\left\|\xi^{n}\left(\boldsymbol{x}^{n}\right)\right\|^{2}
$$

with expectation $E\{\Lambda\}=a P^{3}$. The AIR obtained with the optimized source is given by (6) and can be expressed as

$$
I_{\eta}(\boldsymbol{X} ; \boldsymbol{Y})=\log _{2}\left(1+\frac{P}{\sigma_{n}^{2}+\sigma_{\xi}^{2}\left(\gamma_{\lambda}\right)}\right)-\frac{1}{n} \log _{2} \frac{1}{\eta\left(\gamma_{\lambda}\right)}
$$

where we have made explicit the dependence on the threshold $\gamma_{\lambda}$ of both the acceptance rate $\eta\left(\gamma_{\lambda}\right)$ and the NLI variance (after selection) $\sigma_{\xi}^{2}\left(\gamma_{\lambda}\right)$, and we have assumed that $E\left\{|Y|^{2}\right\}=P+\sigma_{n}^{2}+\left.\sigma_{\xi}^{2}\left(\gamma_{\lambda}\right)\right|^{2}$ By reducing $\gamma_{\lambda}$, we accept only sequences with a lower NLI, reducing $\sigma_{\xi}^{2}\left(\gamma_{\lambda}\right)$. At the same time, we accept less sequences, reducing also $\eta\left(\gamma_{\lambda}\right)$. In terms of AIR, the first effect is beneficial, whereas the second one is detrimental. The opposite behavior is obtained by increasing $\gamma_{\lambda}$. Two special cases are obtained for $\gamma_{\lambda} \rightarrow 0$ and $\gamma_{\lambda} \rightarrow \infty$. In the first case, $\eta \rightarrow 0$ and the information rate vanishes (no sequences are available). In the second case, $\eta \rightarrow 1$, the source remains unbiased, and $\sigma_{\xi}^{2}\left(\gamma_{\lambda}\right) \rightarrow a P^{3}$, so that 17 reduces to (15).

In order to study the more general case and find the optimal threshold $\gamma_{\lambda}$ that maximizes the AIR, we need to make some additional assumptions about the NLI distribution. In particular, we assume that when the input is unbiased (i.i.d. Gaussian symbols), the cost function in (16) has a gamma

\footnotetext{
${ }^{2}$ In practice, we assume that the output power equals the input power plus the noise and NLI power, implying that the three processes are uncorrelated. This is clearly a simplification, since NLI does depend on the input symbols. Nonetheless, the following analysis would produce qualitatively similar results by making more realistic assumptions, e.g., $E\left\{|Y|^{2}\right\}=P+\sigma_{n}^{2}$ (the total energy is preserved by the nonlinear propagation), or $E\left\{|Y|^{2}\right\}=P+\sigma_{n}^{2}-$ $\sigma_{\xi}^{2}\left(\gamma_{\lambda}\right)$ (the NLI is anticorrelated with the signal and reduces the output power, for instance due to the loss induced by spectral broadening).
}

distribution with shape parameter $n^{\prime} \leq n$ and expectation $E\{\Lambda\}=a P^{3}$

$$
p_{\lambda}(\lambda)=\frac{1}{\Gamma\left(n^{\prime}\right)}\left(\frac{n^{\prime}}{a P^{3}}\right)^{n^{\prime}} \lambda^{n^{\prime}-1} \exp \left(-\frac{n^{\prime} \lambda}{a P^{3}}\right), \quad \lambda>0
$$

where $\Gamma(n)$ is the gamma function [58, eq. (6.1.1)]. This assumption is motivated by the observation that, if NLI were indeed statistically equivalent to AWGN, as suggested by the GN model, the distribution of the cost function would be exactly the one in $(18)$ but with shape $n^{\prime}=n$-i.e., a rescaled chi-squared distribution with $2 n$ degrees of freedom. On the other hand, adjacent NLI samples are not really independent, as the same input symbols are involved in the generation of several consecutive NLI samples, so that it is reasonable to assume that the actual shape parameter in (18) might be reduced. Again, (18) is just a working assumption, with no claims of accuracy. In the next section, more realistic fiber models will be studied numerically, also showing how far the assumptions of this section are from reality.

The dependence of the acceptance rate and variance on the threshold can be computed from the distribution in (18). The acceptance rate equals the cumulative distribution function (cdf)

$$
\eta\left(\gamma_{\lambda}\right)=\int_{0}^{\gamma_{\lambda}} p_{\lambda}(\lambda) d \lambda=\frac{\gamma\left(n^{\prime}, n^{\prime} \gamma_{\lambda} P^{-3} / a\right)}{\Gamma\left(n^{\prime}\right)}
$$

where $\gamma(s, x)$ is the lower incomplete gamma function with parameter $s$ [58, eq. (6.5.2)]. The NLI variance equals the conditional expectation

$$
\begin{aligned}
\sigma_{\xi}^{2}\left(\gamma_{\lambda}\right) & =E\left\{\Lambda \mid \Lambda<\gamma_{\lambda}\right\}=\int_{0}^{\gamma_{e}} \lambda \frac{p_{\lambda}(\lambda)}{\eta\left(\gamma_{\lambda}\right)} d \lambda \\
& =\frac{a P^{3}}{n^{\prime}} \frac{\gamma\left(n^{\prime}+1, n^{\prime} \gamma_{\lambda} P^{-3} / a\right)}{\gamma\left(n^{\prime}, n^{\prime} \gamma_{\lambda} P^{-3} / a\right)}
\end{aligned}
$$

The AIR is eventually obtained by replacing $(19)$ and $(20)$ in (17)

$$
\begin{aligned}
I_{\eta}(\boldsymbol{X} ; \boldsymbol{Y})= & \log _{2}\left(1+\frac{P}{\sigma_{w}^{2}+\frac{a P^{3}}{n^{\prime}} \frac{\gamma\left(n^{\prime}+1, n^{\prime} \gamma_{\lambda} P^{-3} / a\right)}{\gamma\left(n^{\prime}, n^{\prime} \gamma_{\lambda} P^{-3} / a\right)}}\right) \\
& -\frac{1}{n} \log _{2} \frac{\Gamma\left(n^{\prime}\right)}{\gamma\left(n^{\prime}, n^{\prime} \gamma_{\lambda} P^{-3} / a\right)}
\end{aligned}
$$

Interestingly, while the Gaussian AIR (15) has the typical peaky behavior that is commonly found for most available capacity lower bounds for the nonlinear optical fiber-it reaches a peak at some optimal power and then decays again to zero at high power-this is not necessarily the case for the AIR 21] with sequence selection. In fact, by setting the threshold to the approximately optimal value

$$
\gamma_{\lambda}^{\text {opt }} \approx \frac{n^{\prime}+1}{n-n^{\prime}} \sigma_{n}^{2}
$$

three different asymptotic behaviors can be observed for $P \rightarrow \infty$ : i) for $n^{\prime}<n / 3$, the AIR grows unbounded as $\log _{2}\left(P^{1-3 n^{\prime} / n}\right)$; ii) for $n^{\prime}=n / 3$, the AIR saturates to a finite constant value; for $n^{\prime}>n / 3$, the AIR vanishes after reaching a finite peak at some optimum power (as in the unshaped case). 
This result, obtained specifically for the distribution in (18), is more general and holds whenever the cdf in (19) grows as $\sim \gamma_{\lambda}^{n^{\prime}}$ for small $\gamma_{\lambda}$. A slow growth $\left(n^{\prime}<n / 3\right)$ implies that the AIR can always be increased by increasing the power $P$ and simultaneously decreasing the selection threshold $\gamma_{\lambda}$ to keep the NLI limited, since the rate loss caused by the selection is more than compensated by the corresponding increase of the SNR. On the other hand, this is not possible, at least asymptotically, when the growth is faster $\left(n^{\prime}>n / 3\right)$.

Fig. 4 shows the AIR (17) with optimized selection threshold for the block-memoryless nonlinear channel (14) with nonlinear coefficient $a=0.01$, noise variance $\sigma_{w}^{2}=0.001$, block length $n=60$, under the assumption that the NLI intensity, averaged over the block, has the distribution in (18) with different values of the scale parameter $n^{\prime}$. For comparison, the figure reports also the linear channel capacity and the Gaussian AIR (15). As anticipated, three fundamentally different asymptotic behaviors (unbounded growth, saturation, or decay to zero) are observed, depending on $n^{\prime}$. Even in the worst case $\left(n^{\prime}=30>n / 3\right)$, in which the AIR decays to zero for $P \rightarrow \infty$, a gain with respect to the unshaped case is obtained in the nonlinear regime.

\section{Numerical RESUlts}

\section{A. System Description}

The system is depicted in Fig 1, while the scenario and link parameters are the same considered in [25], [27]-a $1000 \mathrm{~km}$ standard single-mode fiber link with attenuation $\alpha=0.2 \mathrm{~dB} / \mathrm{km}$, group-velocity-dispersion parameter $\beta_{2}=$ $21.7 \mathrm{ps}^{2} / \mathrm{km}$, and nonlinear coefficient $\gamma=1.27 \mathrm{~W}^{-1} \mathrm{~km}^{-1}$; ideal distributed amplification with unitary spontaneous emission coefficient; and, unless otherwise specified, five dualpolarization 50 GBd Nyquist-WDM channels with sinc pulse shape and $50 \mathrm{GHz}$ spacing ${ }^{3}$ The transmitted symbols are i.i.d. circularly symmetric Gaussian variables (unbiased distribution), further processed by the rejection sampling machine in Fig. 3 when sequence selection is applied (optimized distribution). At the receiver, the central WDM channel is demultiplexed by using an ideal rectangular filter with $50 \mathrm{GHz}$ bandwidth and further processed according to the selected DSP (ideal dispersion compensation or ideal single-channel DBP) and decoding metric (optimized for the AWGN or PPN channel). When subcarrier multiplexing is considered, each WDM channel is divided into four $12.5 \mathrm{GBd}$ subcarriers with sinc pulse shape and $12.5 \mathrm{GHz}$ spacing. Before detection, a possible constant average phase rotation affecting the signal (due to nonlinearity) is estimated and removed.

Fiber propagation is emulated by using the split step Fourier method (SSFM); a step size of $100 \mathrm{~m}$ and a sampling rate of $400 \mathrm{GHz}$ (eight samples per symbol in the single-carrier case) are used for the WDM scenario; a step size of $500 \mathrm{~m}$ and a sampling rate of $100 \mathrm{GHz}$ (two samples per symbol in the single-carrier case) for the single-channel scenario, for the

\footnotetext{
${ }^{3}$ The same scenario was considered also in $[1]$, though we accidentally used a slightly higher nonlinear coefficient $\gamma=1.3 \mathrm{~W}^{-1} \mathrm{~km}^{-1}$, which explains why the AIR values in [1] are slightly lower in the nonlinear regime.
}

computation of the cost function, and for the implementation of DBP.

The results of this section are shown in terms of achievable $\mathrm{SE}$, measured in bits/s/Hz/pol and estimated by the procedure described in Section III-C with $N=2^{18}$ symbols. As a benchmark, all the figures report also the SE obtained on the nonlinear channel when the system is optimized in the absence of nonlinear effects, i.e., for a system with ideal dispersion compensation, i.i.d. Gaussian input symbols, and AWGN detection. The capacity per unit bandwidth for the linear channel $C=\log _{2}(1+\mathrm{SNR})$ is also reported as a reference.

\section{B. Single-Channel Single-Polarization System}

The first numerical test is performed in a simple scenario, considering a single-polarization single-channel system with ideal dispersion compensation. The main aim of this test is to study the behavior of the proposed strategy in a more realistic channel, where intrachannel NLI (including signal-noise interaction and spectral broadening) is accurately modeled by the SSFM, possibly departing from the assumptions made for the analytic study in Section IV (block-memoryless, cubic scaling with power, gamma distribution). Moreover, the test will also show how far those assumptions are from reality, and how fast the cdf of the NLI energy grows with respect to the critical rate defined in Section IV

In this scenario, we employ the simple cost function in (13), without averaging over the realizations. In practice, the selection is implemented by the following fast procedure:

- generate a very long input sequence $\boldsymbol{x}^{N^{\prime}}$ by drawing samples from the unbiased distribution and evaluate the corresponding output sequence $\hat{\boldsymbol{y}}^{N^{\prime}}$ by SSFM propagation through the noiseless channel;

- take all the $N_{p}=N^{\prime}-n+1$ subsequences of length $n$, $\left\{\boldsymbol{x}_{i+1}^{i+n}\right\}$, for $i=0, \ldots, N_{p}-1$, that are contained in $\boldsymbol{x}^{N^{\prime}}$ as proposed sequences;

- for each proposed sequence, compute the cost function

$$
\lambda\left(\boldsymbol{x}_{i+1}^{i+n}\right)=\left\|\hat{\boldsymbol{y}}_{i+1}^{i+n}-\boldsymbol{x}_{i+1}^{i+n}\right\|^{2}
$$

- accept the $N_{a}$ subsequences for which the cost function is below threshold.

If more sequences are needed, the procedure is repeated several times. The selection is performed at a single launch power (near the optimum launch power without selection), under the hypothesis that the ranking induced by the cost function on the sequences does not change significantly with power. This is true, for instance, if the NLI scales cubically with power. The AIR and SE at the desired launch power are eventually estimated by using the procedure described in Section III-C, where the input sequence $\boldsymbol{x}^{N}$ is formed by concatenating the accepted sequences.

First, we compare the assumptions made in Section IV about the simplified nonlinear channel (14) with the actual NLI measured in the more realistic channel considered here. Fig. 5 (a) shows the distribution of the cost function (13), corresponding to the NLI energy per symbol, evaluated for 
$N_{p} \approx 1.35 \times 10^{9}$ proposed sequences with blocklength $n=256$ and normalized to the mean signal energy per symbol. The empirical distributions obtained at two different powers (one rescaled by the cube of the power ratio to match the other) are compared to the gamma distribution (18) with $n^{\prime}=n$ that would be obtained for the energy of an AWGN process. The two empirical distributions are practically superimposed, confirming the assumption that the NLI scales with very good approximation with the cube of the input power. This is even better illustrated by the inset of Fig. 5(a), where a point is reported for each tested sequence, with coordinates equal to the NLI obtained for the two different launch powers. As expected, all the points practically lie on the straight line $y=8 x$. Moreover, the empirical distributions of the NLI energy are significantly different and wider than the distribution that would be obtained if NLI were statistically equivalent to an AWGN, meaning that the AWGN assumption typically made in GN models is too pessimistic and not accurate enough for this approach. This is even better appreciated in Fig. 5.b), which reports the left tails of the empirical cdf obtained for different values of the block length $n$. The results show that the particular characteristics of the generated NLI and the dependence between its samples make its cdf grow with an exponent that is not only lower than the value $n$ that would be obtained for AWGN, but also much lower than the highest exponent $n / 3$ that guarantees an unbounded growth of AIR with power according to the theory developed in Section IV This is clearly not a proof that the same unbounded growth can be actually obtained on this more realistic channel, but at least a good reason to expect a significant AIR improvement.

As explained in Section III by setting a threshold on the cost function (the horizontal axis in Fig. 5), it is possible to select only the "good" sequences that cause a lower NLI; by reducing the threshold (moving it to the left), both the acceptance rate (which equals the cdf) and the average NLI energy decrease. The SE as a function of the acceptance rate is reported in Fig. 6.a) for different values of the block length $n$ and two different launch powers: slightly more than the optimal launch power without sequence selection $(-9 \mathrm{dBm})$ and $1 \mathrm{~dB}$ more. The SE increases monotonically for large $n$ (256 and 1024). A similar behavior could be expected for $n=64$, given that the corresponding cdf in Fig. 5(b) grows more slowly than $\sim \lambda^{n / 3}$. However, the theory in Section IV and the cost function (13) are based on the assumption of a block-memoryless NLI, and do not account for inter-block NLI. In fact, the value of the cost function computed for a specific sequence by the above procedure depends also on the adjacent sequences, meaning that a low-cost sequence selected in this way might, in fact, have a higher cost when combined with different adjacent sequences. The impact of inter-block NLI affects mostly the symbols at the edges of the sequence, so that it becomes less and less relevant as $n$ increases. In principle, $n$ should be taken as large as possible to obtain the best performance. However, as can be inferred from (19) and (22) and from Fig. 5(b), a longer $n$ requires a lower acceptance rate to achieve the optimal performance, which means testing many more sequences. The lowest acceptance rate for which we were able to obtain reliable results $\left(N_{a}>1000\right.$ accepted sequences) in this scenario is $\sim 10^{-6}$. At this rate, we have obtained the highest gain for $n=256$. However, a longer $n$ should offer a higher potential gain, provided that a sufficiently lower acceptance rate could be achieved. This is indeed suggested by the behavior of the curve for $n=1024$. An alternative approach to limit the impact of inter-block NLI without increasing $n$ is the use of the more accurate cost function (12), as it will be shown in Section $\mathrm{V}-\mathrm{C}$

Fig. 6 also shows that without sequence selection (or for a high acceptance rate) the lower launch power of $-9 \mathrm{dBm}$ offers the best performance, as we are already beyond the optimal power. However, when decreasing the acceptance rate, the SE curves at higher power cross the ones at lower power, as the optimal launch power increases. In general, to fully exploit the potential of the sequence selection approach, it is necessary to simultaneously decrease the acceptance rate and increase the launch power. In fact, by reducing the acceptance rate, we select sequences that cause less NLI, so that we can increase their launch power and hence improve the SNR.

Fig. 6(b) shows the SE as a function of the launch power for a block length $n=256$ and different values of the acceptance rate. As expected, reducing the acceptance rate improves the SE, not only at the launch power at which the sequences have been selected, but in the whole nonlinear regime. As a result, both the optimal launch power and the peak SE increase by reducing the acceptance rate. Clearly, a lower acceptance rate means also a higher rate loss, which explains why the SE in the linear regime is slightly lower. The results in Fig. 6(a) and (b) suggest that an even higher SE could be expected by further reducing the acceptance rate and increasing the launch power for $n \geq 256$.

\section{WDM Dual-Polarization System}

The second test is performed in the dual-polarization WDM scenario, where each channel is affected by both inter- and intrachannel NLI. In this case, we consider two different procedures to compute the cost function and select the best sequences. The first one is the fast procedure described in Section $\mathrm{V}-\mathrm{B}$, simply extended to consider two polarizations. The second one, on the other hand, considers the more accurate cost function (12) to average out the impact of inter-block NLI (neglected by the first approach) and will be hence referred to as averaged procedure. This should ensure that the cost of the selected sequences is actually low, regardless of the adjacent sequences that are actually transmitted. In both approaches, the cost function is evaluated in a single-channel noiseless scenario, therefore preferring those sequences that cause low intrachannel NLI, regardless of their impact in terms of interchannel NLI and signal-noise interaction. The averaged procedure is implemented by the following steps:

- generate a very long input sequence $x^{N^{\prime}}$ by drawing samples from the unbiased distribution and evaluate the corresponding output sequence $\hat{\boldsymbol{y}}^{N^{\prime}}$ by SSFM propagation through the noiseless channel;

- take as proposed sequences the $N_{p}=N^{\prime} /\left(n+N_{g}\right)$ disjoint subsequences $\left\{\boldsymbol{x}_{i\left(n+N_{g}\right)+1}^{i\left(n+N_{g}\right)+n}\right\}$, for $i=0, \ldots, N_{p}-1$, 


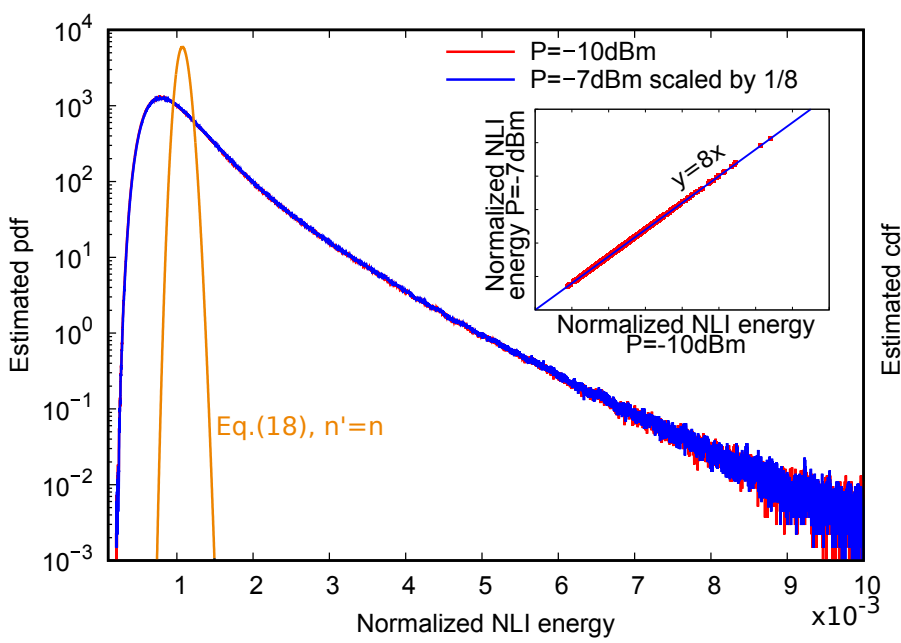

(a)

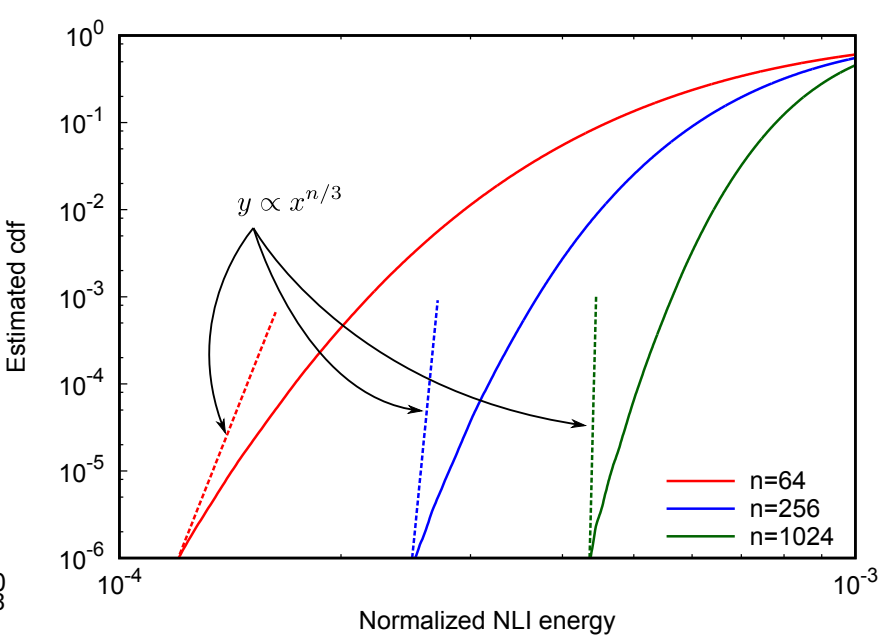

(b)

Fig. 5. Distribution of the normalized NLI energy in the single-channel single-polarization system: (a) empirical distribution for $n=256$ and two different powers (the coordinates of the points in the inset correspond to the realizations obtained for each sequence and the two power values) compared to the gamma distribution 18 with $n^{\prime}=n$; (b) empirical cdf for different blocklength $n$ compared to the power law $\sim \lambda^{n / 3}$ needed for an unbounded growth of the AIR.

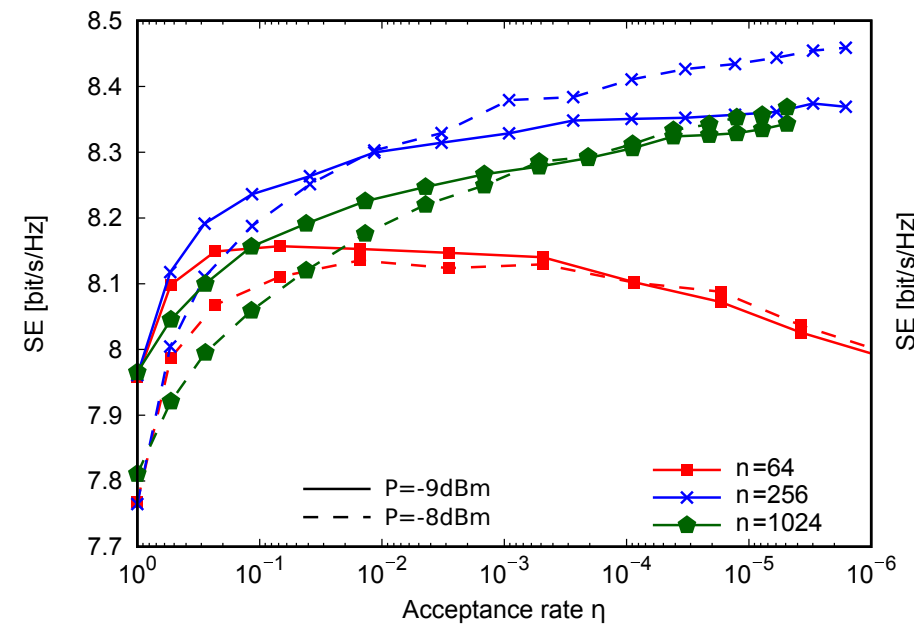

(a)

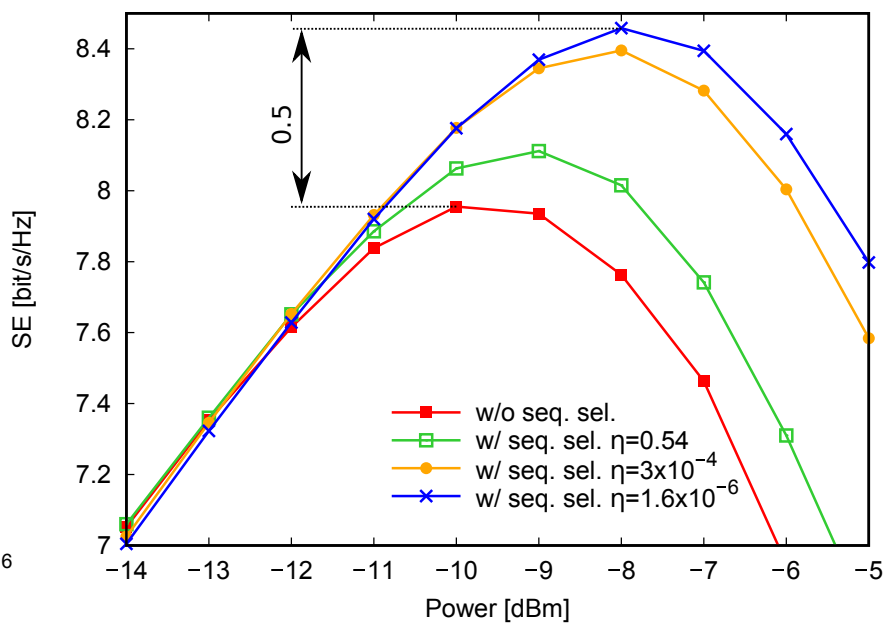

(b)

Fig. 6. Achievable SE for the single-channel single-polarization system with sequence selection: (a) SE versus acceptance rate for different values of the block length $n$ and launch power; (b) SE versus launch power for $n=256$ and different values of the acceptance rate.

that are obtained by dividing $\boldsymbol{x}^{N^{\prime}}$ into subsequences of length $n$, separated by $N_{g}$ guard symbols;

- for each proposed sequence, compute the temporary cost function

$$
\lambda\left(\boldsymbol{x}_{i\left(n+N_{g}\right)+1}^{i\left(n+N_{g}\right)+n}\right)=\left\|\hat{\boldsymbol{y}}_{i\left(n+N_{g}\right)+1}^{i\left(n+N_{g}\right)+n}-\boldsymbol{x}_{i\left(n+N_{g}\right)+1}^{i\left(n+N_{g}\right)+n}\right\|^{2}
$$

- repeat $N_{\text {it }}$ times the previous steps by leaving the proposed subsequences in $\boldsymbol{x}^{N^{\prime}}$ unchanged, while randomly changing all the guard symbols;

- for each proposed sequence, compute the final cost function by averaging the temporary cost functions over the $N_{\text {it }}$ realizations;

- accept the $N_{a}$ subsequences for which the final cost function is below the desired threshold.

If more sequences are needed, the procedure is repeated several times. Also in this case, the selection is performed at a single launch power. The AIR and SE are then estimated on the central channel by following the procedure described in Section III-C, where the sequences of length $N$ transmitted on the five WDM channels are independently generated by concatenating in a random order the selected subsequences of length $n$.

Fig. 7 shows the SE obtained in four different cases: the benchmark without sequence selection, optimized for the linear regime; the system with sequence selection, with $n=256$ dual-polarization symbols, $N_{p}=65512$ and $N_{p}=38350$ for the fast and averaged optimization, respectively, and $\eta=$ 0.002 (chosen as a good trade-off between performance and computational complexity); the system with single-channel ideal DBP and without sequence selection; and the system with both DBP and sequence selection, where the same sequences selected without DBP are transmitted. Sequence selection is performed by using either of the two described procedures to estimate the cost functions (13) or (12), the 


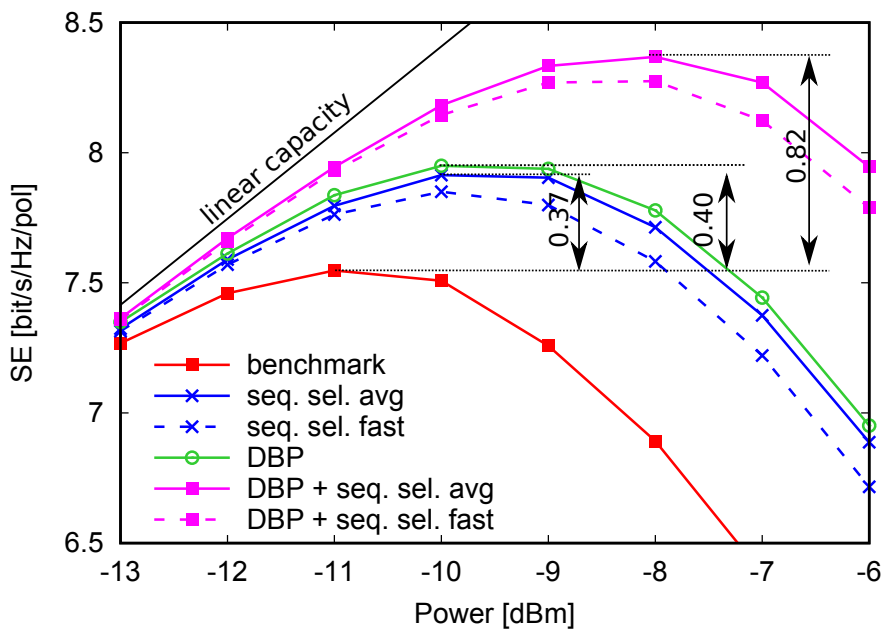

Fig. 7. Achievable SE for the dual-polarization WDM system with sequence selection ( $n=256, \eta=0.002$, and two different cost functions), possibly combined with DBP.

latter by considering $N_{g}=800$ guard symbols and averaging over $N_{\text {it }}=20$ realizations. As anticipated, the second cost function accounts for the average impact of inter-block NLI, providing a more accurate selection and a higher SE gain. With the improved cost function, sequence selection alone yields an SE gain of $0.37 \mathrm{bits} / \mathrm{s} / \mathrm{Hz} / \mathrm{pol}$ with respect to the benchmark. By comparison, DBP yields only a slightly higher gain of $0.39 \mathrm{bits} / \mathrm{s} / \mathrm{Hz} / \mathrm{pol}$, while the combination of the two techniques yields a higher total gain of $0.82 \mathrm{bits} / \mathrm{s} / \mathrm{Hz} / \mathrm{pol}$. This means that the input distribution provided by sequence selection, though optimized to reduce intrachannel NLI, partly mitigates also interchannel NLI, so that it provides an additional gain even when combined with DBP (which completely removes intrachannel NLI).

\section{Combination of Shaping, DBP, and Improved Detection}

As a final test, we combine the proposed sequence selection technique for the optimization of the input distribution, with both DBP and an improved decoding metric optimized for a channel with phase and polarization noise (PPN). The aim is to maximize the achievable SE to improve on the tightest available capacity lower bounds for the WDM channel [25], [27]. In particular, by following [25], we consider a random-walk model for the phase and polarization evolution of the PPN metric, and divide each WDM channel into four subcarriers to obtain the best trade-off between the temporal and frequency coherence of the PPN processes over each subcarrier. Moreover, by following [27], we optimize the power allocation per subcarrier to maximize the SE. The SE with PPN decoding is estimated by using the general procedure described in Section III-C and computing the decoding metric by using the particle filtering approach described in [25], [59].

Fig. 8 shows the new SE lower bound obtained in this way (blue), compared to lower bound [27] (yellow) and to the SE obtained with other configurations. Sequence selection is performed according to the same procedure described in Section $\mathrm{V}-\mathrm{C}$ and based on the cost function $(12)$, considering $n=512$ dual-polarization symbols, $\eta=0.004, N_{p}=47820$,
$N_{g}=800$, and $N_{\text {it }}=20$. The only difference with respect to Section $\mathrm{V}-\mathrm{C}$ is that each proposed subsequence of $n$ symbols is formed by taking $n / 4$ symbols simultaneously transmitted on each subcarrier, and different subsequences are separated by $N_{g} / 4$ guard symbols on each subcarrier. Therefore, as in Section $\mathrm{V}-\mathrm{C}$, the resulting input distribution is optimized for the single-channel noiseless scenario and does not account for the presence of interchannel NLI, for the optimized power allocation per subcarrier, and for the use of DBP and of an improved decoding metric. This allows to keep the computational complexity of the selection process low, but provides a suboptimal input distribution. Nonetheless, even in this case the sequence selection approach provides an additional SE gain when combined with DBP, PPN detection, and optimized power allocation, meaning that the selected sequences are good also for this more complex scenario, though not specifically optimized for it. In practice, DBP removes intrachannel NLI, while both PPN detection and sequence selection mitigate interchannel NLI.

The additional gain of $0.11 \mathrm{bit} / \mathrm{s} / \mathrm{Hz} / \mathrm{pol}$ provided by sequence selection is significantly smaller than in the case studied in Section V-C, but still sufficient to bring the overall gain to $1.4 \mathrm{bit} / \mathrm{s} / \mathrm{Hz} /$ pol with respect to the benchmark, surpassing by $0.04 \mathrm{bit} / \mathrm{s} / \mathrm{Hz} / \mathrm{pol}$ the lower bound in [27]. We remark that the latter uses a slightly different model for PPN evolution; a whitening filter to account also for the correlation of the additive noise; and optimized delays between subcarriers and between WDM channels. These differences explain the slightly higher SE of [27] (yellow curve) with respect to our SE without sequence selection (purple curve). Nonetheless, the optimization of the input distribution based on the sequence selection approach with the suboptimal cost function (12) is more effective than all these techniques, providing a higher SE gain. We expect that our bound could be only slightly improved by including also those techniques. On the other hand, we expect that a more relevant SE gain should be obtained by improving the selection procedure-e.g., by increasing $n$ and $N_{\text {it }}$, decreasing $\eta$, and tailoring the cost function (10) to the WDM scenario and to the considered system configuration.

\section{CONCLUSION}

We have presented a new technique to optimize the input distribution and lower-bound the capacity of the optical fiber channel in a very general setting. The technique uses a rejection sampling algorithm, driven by a properly defined cost function, to generate the sequences of input symbols that are most suitable for the nonlinear channel and the selected decoding metric. The corresponding AIR is then estimated by Monte Carlo averaging. The rate loss induced by the selection process is simply related to the acceptance rate of the rejection sampling algorithm and removed from the AIR.

We have tested the proposed techniques in a few different scenarios. In a simplified optical channel with blockmemoryless NLI, the AIR obtained with the selection process can be computed analytically, showing that an unbounded growth of capacity with power is indeed possible, provided that certain conditions on the distribution of the NLI hold. 


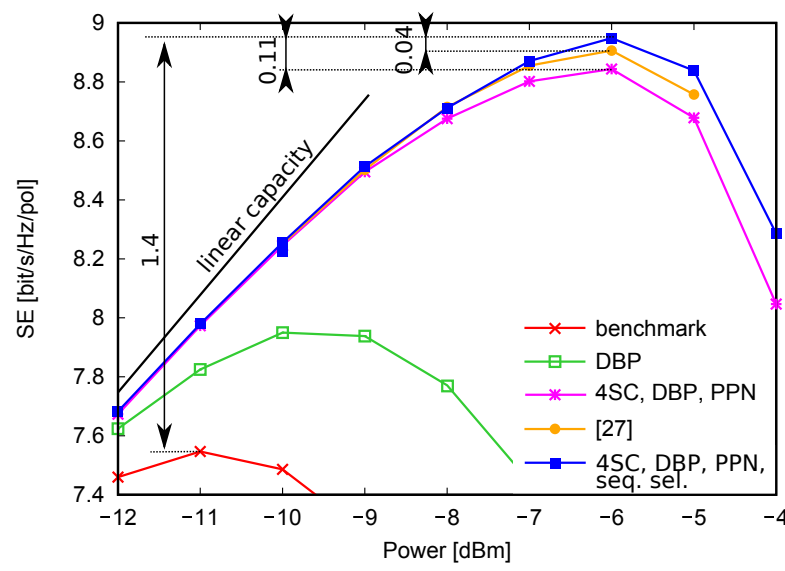

Fig. 8. Achievable SE for the dual-polarization WDM system with sequence selection ( $n=512, \eta=0.004$, averaged cost function) combined with single-channel DBP, subcarrier multiplexing, per-subcarrier power optimization, and PPN detection.

In a still simple but more realistic single-polarization singlechannel scenario, numerical simulations demonstrate a significant improvement of the AIR (and of the corresponding $\mathrm{SE})$. The same simulations also reveal that the above mentioned conditions on the NLI distribution are practically met, suggesting that further AIR improvements are possible by increasing the power and reducing the acceptance rate. In a dual-polarization WDM scenario, the proposed technique yields an AIR gain that is comparable to the gain offered by ideal single-channel DBP; the gain is almost doubled when the two techniques are combined, and further increases when also subcarrier multiplexing and a PPN decoding metric are employed. The peak AIR obtained by the combined techniques exceeds the highest capacity lower bound available in the literature for the same channel, effectively establishing a new lower bound. These results suggest that nonlinear constellation shaping, if properly optimized in a high-dimensional space, can be a valid replacement or an effective complement to DBP and other complex mitigation strategies.

The definition of a suitable cost function is essential for the correct optimization of the input distribution. We have proposed a general definition, related to the minimization of the mismatched conditional entropy; a more specific definition, suitable for intrachannel NLI and an AWGN decoding metric; and a simplified definition, suitable when intrachannel NLI is block-memoryless. Both the specific and the simplified definitions have been implemented and tested numerically. They work well in all the considered scenarios, even beyond the original scope for which they are designed; in fact, the sequences selected in this way mitigate also the impact of interchannel NLI, even when combined with an improved decoding metric. The best performance is obtained with the more complex cost function, whereas the simplified one reduces significantly the computation cost of the selection procedure.

We expect that the numerical results and bounds obtained in this work can be further improved by following different possible approaches. The first one is merely a brute-force approach. The values used for $n, N_{\mathrm{it}}$, and $\eta$ are not optimal, but simply selected as a trade-off between performance and computational cost; thus, better results can be obtained by employing more computational resources to increase $n$ and $N_{\text {it }}$ and to reduce $\eta$. A more interesting approach is that of modifying the cost function. In fact, for simplicity, the input sequences have been simply optimized to minimize intrachannel NLI; higher gains are expected by better tailoring the cost function to the relevant impairments and adopted decoding metric, e.g., accounting also for interchannel NLI and for the use of DBP and PPN decoding. An indication in this sense is provided by 10 . The same approach can be followed also to extend the application of the proposed techniques to other channels and scenarios.

\section{ACKNOWLEDGMENT}

The authors would like to thank Francisco Javier GarcíaGómez for a useful discussion on the comparison between the capacity lower bounds in this work and in [27].

\section{REFERENCES}

[1] M. Secondini, S. Civelli, and E. Forestieri, "Tailored shaping, improved detection, simpler backpropagation: the road to nonlinearity mitigation," in Proc. Eur. Conf. Opt. Commun. (ECOC), Sep. 2021.

[2] G. P. Agrawal, Nonlinear Fiber Optics, 3rd ed. San Diego, CA: Academic Press, 2001.

[3] A. D. Ellis, Z. Jian, and D. Cotter, "Approaching the non-linear Shannon limit," J. Lightw. Technol., vol. 28, no. 4, pp. 423-433, Feb. 2010.

[4] E. Agrell, M. Karlsson, A. R. Chraplyvy, D. J. Richardson, P. M. Krummrich, P. Winzer, K. Roberts, J. K. Fischer, S. J. Savory, B. J. Eggleton, M. Secondini, F. R. Kschischang, A. Lord, J. Prat, I. Tomkos, J. E. Bowers, S. Srinivasan, M. Brandt-Pearce, and N. Gisin, "Roadmap of optical communications," J. Optics, vol. 18, no. 6, p. 063002, 2016.

[5] M. Secondini and E. Forestieri, "Scope and limitations of the nonlinear Shannon limit," J. Lightw. Technol., vol. 35, no. 4, pp. 893-902, Feb. 2017.

[6] A. Splett, C. Kurtzke, and K. Petermann, "Ultimate transmission capacity of amplified optical fiber communication systems taking into account fiber nonlinearities," in Proc. Eur. Conf. Opt. Commun. (ECOC), vol. 2, 1993, pp. 41-44.

[7] P. P. Mitra and J. B. Stark, "Nonlinear limits to the information capacity of optical fiber communications," Nature, vol. 411, no. 6841, pp. 10271030, June 2001.

[8] J. Tang, "The channel capacity of a multispan DWDM system employing dispersive nonlinear optical fibers and an ideal coherent optical receiver," J. Lightw. Technol., vol. 20, no. 7, pp. 1095-1101, July 2002.

[9] K. S. Turitsyn, S. A. Derevyanko, I. V. Yurkevich, and S. K. Turitsyn, "Information capacity of optical fiber channels with zero average dispersion," Phys. Rev. Lett., vol. 91, no. 20, Nov. 2003.

[10] J. M. Kahn and K.-P. Ho, "Spectral efficiency limits and modulation/detection techniques for DWDM systems," IEEE J. Sel. Topics Quantum Electron., vol. 10, no. 2, pp. 259-272, Mar./Apr. 2004.

[11] I. Djordjevic, B. Vasic, M. Ivkovic, and I. Gabitov, "Achievable information rates for high-speed long-haul optical transmission," J. Lightw. Technol., vol. 23, no. 11, pp. 3755-3763, Nov. 2005.

[12] M. H. Taghavi, G. C. Papen, and P. H. Siegel, "On the multiuser capacity of WDM in a nonlinear optical fiber: Coherent communication," IEEE Trans. Inf. Theory, vol. 52, no. 11, pp. 5008-5022, Nov. 2006.

[13] R.-J. Essiambre, G. Kramer, P. J. Winzer, G. J. Foschini, and B. Goebel, "Capacity limits of optical fiber networks," J. Lightw. Technol., vol. 28, no. 4, pp. 662-701, Feb. 2010.

[14] M. I. Yousefi and F. R. Kschischang, "On the per-sample capacity of nondispersive optical fibers," IEEE Trans. Inf. Theory, vol. 57, no. 11, pp. 7522-7541, Nov. 2011.

[15] G. Bosco, P. Poggiolini, A. Carena, V. Curri, and F. Forghieri, "Analytical results on channel capacity in uncompensated optical links with coherent detection," Opt. Exp., vol. 19, no. 26, pp. B438-B449, Dec. 2011.

[16] A. Mecozzi and R.-J. Essiambre, "Nonlinear Shannon limit in pseudolinear coherent systems," J. Lightw. Technol., vol. 30, no. 12, pp. 2011-2024, June 2012. 
[17] R. Dar, M. Shtaif, and M. Feder, "New bounds on the capacity of the nonlinear fiber-optic channel," Opt. Lett., vol. 39, no. 2, pp. 398-401, Jan 2014.

[18] E. Agrell, A. Alvarado, G. Durisi, and M. Karlsson, "Capacity of a nonlinear optical channel with finite memory," J. Lightw. Technol., vol. 32, no. 16, pp. 2862-2876, Aug. 2014.

[19] E. Agrell, "Conditions for a monotonic channel capacity," IEEE Trans. Commun., vol. 63, no. 3, pp. 738-748, Mar. 2015.

[20] G. Kramer, M. I. Yousefi, and F. R. Kschischang, "Upper bound on the capacity of a cascade of nonlinear and noisy channels," in IEEE Information Theory Workshop (ITW), Apr. 2015.

[21] E. Agrell and M. Karlsson, "Influence of behavioral models on multiuser channel capacity," J. Lightw. Technol., vol. 33, no. 17, pp. 3507-3515, Sept. 2015.

[22] E. Agrell, "Capacity bounds in optical communications," in Proc. Eur Conf. Opt. Commun. (ECOC), Sept. 2017.

[23] K. Keykhosravi, G. Durisi, and E. Agrell, "A tighter upper bound on the capacity of the nondispersive optical fiber channel," in Proc. Eur Conf. Opt. Commun. (ECOC), Sept. 2017.

[24] M. Secondini, E. Agrell, E. Forestieri, and D. Marsella, "Fiber nonlinearity mitigation in WDM systems: Strategies and achievable rates," in Proc. Eur. Conf. Opt. Commun. (ECOC), Sept. 2017

[25] M. Secondini, E. Agrell, E. Forestieri, D. Marsella, and M. R. Camara, "Nonlinearity mitigation in WDM systems: Models, strategies, and achievable rates," Journal of Lightwave Technology, vol. 37, no. 10, pp. 2270-2283, 2019.

[26] F. J. García-Gómez and G. Kramer, "Mismatched models to lower bound the capacity of optical fiber channels," Journal of Lightwave Technology, vol. 38, no. 24, pp. 6779-6787, 2020.

[27] — " "Mismatched models to lower bound the capacity of dualpolarization optical fiber channels," Journal of Lightwave Technology, 2021.

[28] M. Sefidgaran and M. Yousefi, "Lower bound on the capacity of the continuous-space SSFM model of optical fiber," arXiv preprint arXiv:2011.11341, 2021.

[29] M. Secondini, "Chapter 20 - information capacity of optical channels," in Optical Fiber Telecommunications VII, A. E. Willner, Ed. Academic Press, 2020, pp. 867-920. [Online]. Available: https: //www.sciencedirect.com/science/article/pii/B9780128165027000233

[30] C. E. Shannon, "A mathematical theory of communication," Bell Syst. Tech. J., vol. 27, no. 3/4, pp. 379-423/623-656, July/Oct. 1948.

[31] F. R. Kschischang and S. Pasupathy, "Optimal nonuniform signaling for Gaussian channels," IEEE Transactions on Information Theory, vol. 39, no. 3, pp. 913-929, 1993.

[32] E. Agrell, G. Durisi, and P. Johannisson, "Information-theory-friendly models for fiber-optic channels: A primer," in IEEE Information Theory Workshop (ITW), Apr. 2015.

[33] M. Secondini and E. Forestieri, "Analytical fiber-optic channel model in the presence of cross-phase modulation," IEEE Photon. Technol. Lett., vol. 24, no. 22, pp. 2016-2019, Nov. 2012.

[34] R. Dar, M. Feder, A. Mecozzi, and M. Shtaif, "Properties of nonlinear noise in long, dispersion-uncompensated fiber links," Opt. Exp., vol. 21, no. 22, pp. $25685-25699$, Nov. 2013

[35] R. Dar and P. J. Winzer, "Nonlinear interference mitigation: methods and potential gain," J. Lightw. Technol., vol. 35, no. 4, pp. 903-930, 2017.

[36] M. Secondini, E. Forestieri, and G. Prati, "Achievable information rate in nonlinear WDM fiber-optic systems with arbitrary modulation formats and dispersion maps," J. Lightw. Technol., vol. 31, no. 23, pp. 38393852, Dec. 2013

[37] M. Secondini and E. Forestieri, "On XPM mitigation in WDM fiberoptic systems," IEEE Photon. Technol. Lett., vol. 26, no. 22, pp. 22522255, 2014

[38] D. Marsella, M. Secondini, E. Agrell, and E. Forestieri, "A simple strategy for mitigating XPM in nonlinear WDM optical systems," in Proc. Opt. Fiber Commun. Conf. (OFC), 2015, paper Th4D-3.

[39] G. Böcherer, F. Steiner, and P. Schulte, "Bandwidth efficient and ratematched low-density parity-check coded modulation," IEEE Transactions on Communications, vol. 63, no. 12, pp. 4651-4665, 2015.

[40] F. Buchali, F. Steiner, G. Böcherer, L. Schmalen, P. Schulte, and W. Idler, "Rate adaptation and reach increase by probabilistically shaped 64 QAM: An experimental demonstration," J. Lightw. Technol., vol. 34 no. 7, pp. 1599-1609, 2016.

[41] Y. C. Gültekin, W. J. van Houtum, A. G. Koppelaar, and F. M. Willems, "Enumerative sphere shaping for wireless communications with shor packets," IEEE Transactions on Wireless Communications, vol. 19, no. 2 , pp. 1098-1112, 2019.
[42] T. Fehenberger, A. Alvarado, G. Böcherer, and N. Hanik, "On probabilistic shaping of quadrature amplitude modulation for the nonlinear fiber channel," J. Lightw. Technol., vol. 34, no. 21, pp. 5063-5073, Nov. 2016.

[43] K. Kojima, T. Yoshida, T. Koike-Akino, D. S. Millar, K. Parsons, M. Pajovic, and V. Arlunno, "Nonlinearity-tolerant four-dimensional 2A8PSK family for 5-7 bits/symbol spectral efficiency," J. Lightw. Technol., vol. 35, no. 8, pp. 1383-1391, 2017.

[44] B. Chen, C. Okonkwo, H. Hafermann, and A. Alvarado, "Polarizationring-switching for nonlinearity-tolerant geometrically shaped fourdimensional formats maximizing generalized mutual information," $J$. Lightw. Technol., vol. 37, no. 14, pp. 3579-3591, 2019.

[45] O. Geller, R. Dar, M. Feder, and M. Shtaif, "A shaping algorithm for mitigating inter-channel nonlinear phase-noise in nonlinear fiber systems," J. Lightw. Technol., vol. 34, no. 16, pp. 3884-3889, Aug. 2016.

[46] T. Fehenberger, H. Griesser, and J.-P. Elbers, "Mitigating fiber nonlinearities by short-length probabilistic shaping," in Optical Fiber Communication Conference. Optical Society of America, 2020, pp. Th1I-2.

[47] J. Cho, X. Chen, G. Raybon, D. Che, E. Burrows, S. Olsson, and R. Tkach, "Shaping lightwaves in time and frequency for optical fiber communication," 2021. [Online]. Available: https: //www.researchsquare.com/article/rs-452651/v1

[48] Y. C. Gültekin, A. Alvarado, O. Vassilieva, I. Kim, P. Palacharla, C. Okonkwo, and F. M. Willems, "Kurtosis-limited sphere shaping for nonlinear interference noise reduction in optical channels," arXiv preprint arXiv:2105.14794, 2021.

[49] S. Civelli, E. Forestieri, and M. Secondini, "Interplay of probabilistic shaping and carrier phase recovery for nonlinearity mitigation," in 2020 European Conference on Optical Communication (ECOC). IEEE, 2020, pp. $1-3$.

[50] S. Civelli, E. Forestieri, A. Lotsmanov, D. Razdoburdin, and M. Secondini, "A sequence selection bound for the capacity of the nonlinear fiber channel," in Proc. Eur. Conf. Opt. Commun. (ECOC), Sep. 2021.

[51] E. Wang and C. R. Menyuk, "Polarization evolution due to the Kerr nonlinearity and chromatic dispersion," J. Lightw. Technol., vol. 17 no. 12 , pp. 2520-2529, Dec. 1999.

[52] J. G. Proakis, Digital Communications, 4th ed. McGraw Hill, 2001.

[53] D. M. Arnold, H.-A. Loeliger, P. O. Vontobel, A. Kavčić, and W. Zeng, "Simulation-based computation of information rates for channels with memory," IEEE Trans. Inf. Theory, vol. 52, no. 8, pp. 3498-3508, Aug. 2006.

[54] R. G. Gallager, Information Theory and Reliable Communication. New York: Wiley, 1968

[55] T. M. Cover and J. A. Thomas, Elements of Information Theory, 2nd ed. Hoboken, NJ: Wiley, 2006.

[56] N. Merhav, G. Kaplan, A. Lapidoth, and S. S. Shitz, "On information rates for mismatched decoders," IEEE Trans. Inf. Theory, vol. 40, no. 6 , pp. 1953-1967, Nov. 1994

[57] P. Poggiolini, "The GN model of non-linear propagation in uncompensated coherent optical systems," J. Lightw. Technol., vol. 30, no. 24, pp. 3857-3879, Dec. 2012.

[58] M. Abramowitz and I. A. Stegun, Handbook of mathematical functions with formulas, graphs, and mathematical tables. US Government printing office, 1972, vol. 55.

[59] J. Dauwels and H.-A. Loeliger, "Computation of information rates by particle methods," IEEE Trans. Inf. Theory, vol. 54, no. 1, pp. 406-409, Jan. 2008 